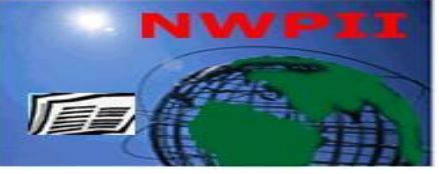

American Journal of Biomedical Sciences

ISSN: 1937-9080

nwpii.com/ajbms

\title{
Scaffold Development and Characterization Using CAD System
}

\author{
Ahmed Farag Aly ${ }^{1 *}$, Ahmed Agameia', Amal Samir Eldesouky ${ }^{1}$, and Mohamed A. Sharaf ${ }^{2}$ \\ ${ }^{1}$ Department of Biomedical Engineering, Helwan University, EGYPT. \\ ${ }^{2}$ Department of Chemistry, Helwan University, EGYPT. \\ *Corresponding Author: \\ Dr. Ahmed Farag Aly \\ Associate Professor \\ Department of Biomedical Engineering \\ Helwan University \\ EGYPT \\ Email: afarag@mcit.gov.eg
}

Received: 19 October 2010; $\mid$ Revised: 9 March 2011; | Accepted: 11 August 2011

\begin{abstract}
Morphology and mechanical properties of scaffolds seeded with osteoblastes cells used for bone and cartilage repair are the critical factors in bone tissue engineering. In this work, adding CMC and controlling temperature for nano-hydroxyapatite (HA)-b-tricalcium phosphate (b-TCP) scaffold using Polymeric sponge method provide suitable properties. A developed computer system was used to determine properties of scaffold. Porosity, shape and connectivity of pores were analysed based on image processing method. Cells were seeded on scaffold and the differentiation rate was calculated using image analysis. The fabricated sample showed high porosity (nearly 61\%) and high compressive strength (nearly $16 \mathrm{MPa}$ ), as well as having a well pore size of $200 \mu \mathrm{m}$ and more. Comparing to Archimedes method, the image result was more accurate. Internal porosity was more than surface porosity due to skin effect.
\end{abstract}

Keywords: Biomaterial, computer aided system, interconnection, porosity, morphology.

\section{Introduction}

Composites biodegradable polymers and bioactive ceramics in the form of biocompatible scaffolds have been proposed and used in the field of tissue engineering with emphasis towards hard tissue regeneration [1]. The generation biomaterials are expected to be degradable and bioactive porous scaffolds for the living bone tissue engineering reconstruction [1]. Scaffold for bone tissue engineering must be biocompatible, osteoconductive, and have a macroporous structure [2]. Hydroxyapatite (HA) bioceramic have been found to bond with living bone tissue through a bone-like apatite layer due to its good biocompatibility, osteoconductivity and a similar mineral composition to natural bone [3]. Due to its brittleness, it isn't recommended for load bearing scaffold. B-TCP is chemically stable and has a fairly fast bioresorption rate [4]. TCP is regarded as an ideal bone substitute. carboxymethyl 
cellulose (CMC) can react strongly with $\mathrm{CS}$ and act as an ionic cross-linking agent at the appropriate $\mathrm{pH}$ [5].

In order to understand the behaviour of scaffold, quantitative knowledge of the morphology and the mechanical behaviour is necessary. Recent advances in computing technologies both in terms of hardware and software have helped in the advancement of design and analysis. Therefore, the porous structures need to be quantified morphologically.

We prepared hydroxylapatite and tri-calcium phosphate nano-particles. Different concentrations of powder were used to get slurry. CMC was added to provide strength. Sponge was immersed in slurry and treated using temperature. Mechanical properties, degradation behaviour and cell proliferation were tested. Morphology was detected using developed computer system. Segmentation is a necessary step. In the past few decades, many effective algorithms have been proposed to perform the computer-aided segmentation. Compared with the algorithms for common image processing, this system is used for porous bio-materials.

Also, images are usually influenced by noises and partial volume effect [6], algorithms should be sophisticated enough to handle the segmentation task. Algorithms are classified into two categories: algorithms based on threshold, algorithms based on morphology operations.

\section{Materials and Methods}

\subsection{Nano-particles Preparation}

The basic materials, hydroxyapatite and tricalcium phosphate particles, were prepared in lab using chemical precipitation method. The precipitates are used to prepare scaffold. CMC is used to strength scaffold and control rate of degradation. Fig. 1 shows the procedure of Nanoparticles preparation.

\subsubsection{Synthesis of Nano hydroxyapatite Particles}

Hydroxyapatite was prepared using chemical precipitation method. $500 \mathrm{ml}$ of $0.6 \mathrm{M}\left(\mathrm{NH}_{4}\right)_{2} \mathrm{PO}_{4}$ were added to DI water. $500 \mathrm{ml}$ of $1 \mathrm{M}$ calcium nitrate solution was prepared by dissolving in DI water. The $\mathrm{pH}$ of the solution was adjusted to 11 by addition of $\mathrm{NH}_{4} \mathrm{OH}$. White precipitates of hydroxyapatite were formed by dropping diammonium hydrogen phosphate solution to calcium nitrate solution under constant stirring for one hour. The solution was reflux for one hour and aged for one day. The precipitates washed with DI water, dried at $80^{\circ} \mathrm{C}$ for a day and calcinated at $800{ }^{\circ} \mathrm{C}$ for one hour.

\subsubsection{Synthesis of Nano -tricalcium Phosphate}

Tricalcium phosphate was prepared using chemical precipitation method. Solution containing $0.4 \mathrm{M}$ of (NH4)2PO4 was added to calcium nitrate solution containing $0.6 \mathrm{M}$ of $\mathrm{Ca}(\mathrm{NO} 3) 2$ _ $4 \mathrm{H} 2 \mathrm{O}$ to make $1000 \mathrm{ml}$ of solution. The $\mathrm{pH}$ of the solution was adjusted to 10 by addition of $\mathrm{NH} 4 \mathrm{OH}$. White precipitates were formed solution aged for one day. The precipitates washed with DI water and ethanol dried at $80{ }^{\circ} \mathrm{C}$ for another day then the temperature increased to $300{ }^{\circ} \mathrm{C}$ for three hours. The dried powder was milled and calcinated at $900{ }^{\circ} \mathrm{C}$ for two hours.

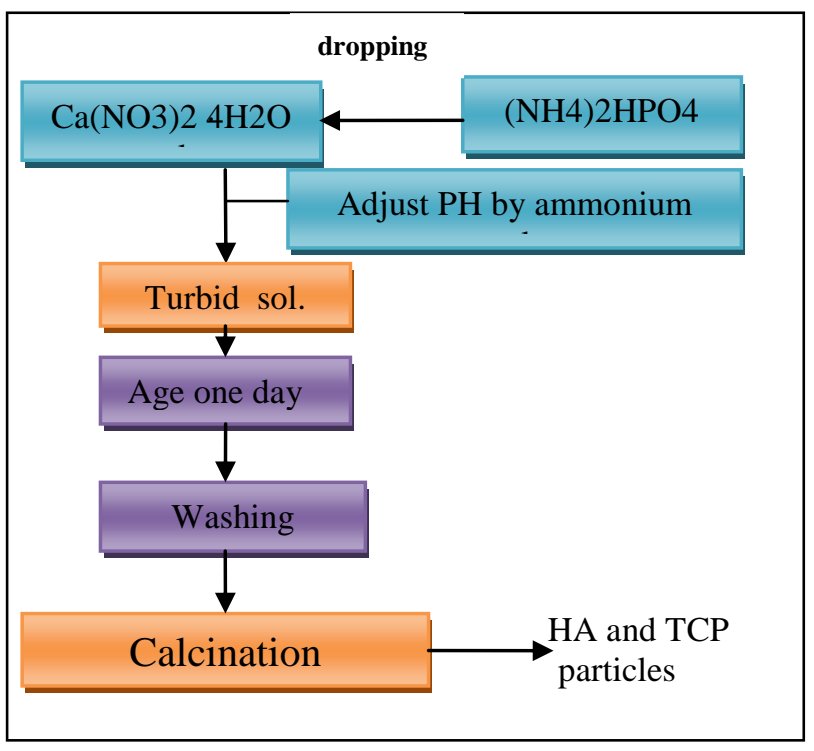

Figure 1. Preparation method of powders.

\subsection{Polymeric Sponge Method}

The polymeric sponge method is performed by impregnating porous polymeric substrates (sponges) with HA/TCP slurry. The slurry was prepared by dispersing calcinated $\mathrm{HA}$ and TCP powders in solution of poly vinyl alcohol (PVA) (5 weight \%) as the binder. Drops of CMC were 
added. The soaked sponge was oven dried at 400C for 24 hours. The dried sponge was sintered at $650 \mathrm{C}$ for 3 hour at a heating rate of $5 \mathrm{C}$ per minute. The samples were kept at $650 \mathrm{C}$ for one hour for binder burn out. The sintered porous scaffold characterized for strength, porosity, pore size distribution and microstructure. The process flow chart for fabrication is shown in Fig. 2.

\subsection{Mechanical Test}

The compressive strength was tested for the three concentrations of HA and b-TCP with a testmetric universal testing machine (AG-10AT, Japan) with a compression strength at rate of 0.5 $\mathrm{mm} / \mathrm{min}$ _at a room temperature of with a relative humidity of 15 percent until $50 \%$ reduction in specimen height.

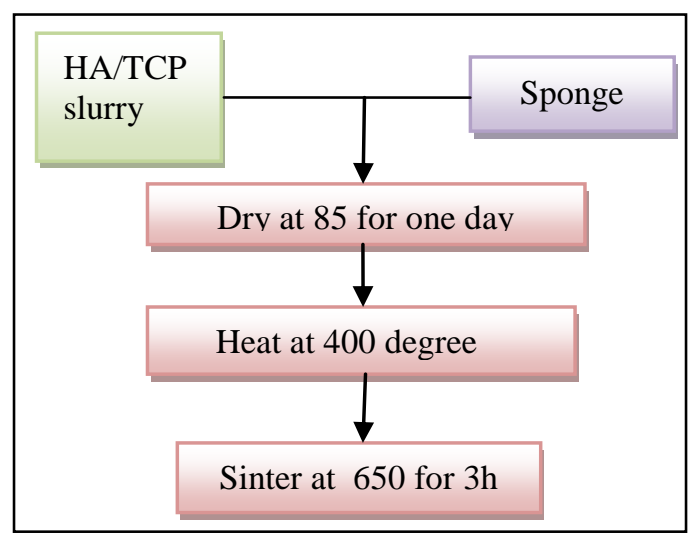

Figure 2. Polymeric sponge method.

The scaffold was cut into blocks with a size of $10 \ldots 12 \mathrm{~mm}$. Three parallel samples were tested for every scaffold and the mean value of the compressive strength of different scaffolds was given. The average data of the test results are plotted. The elastic modulus was calculated as the slope of the initial linear portion.

\subsection{Scaffold and Image Analysis}

Scaffold sample were imaged via scanned electron microscope. The samples were mounted on cupper stubs and coated with gold using coated sputter (S150A Edward, England). The specimens then were examined under JXA-840A electron probe micro analyzer (Jeol,-Japan). Scanning electron microscopy (SEM) was used to image and determine the morphology of the scaffold using our developed system (CAD system).
Binary images of SEM images are obtained using threshold. Segmentation is used for detecting solid area of scaffold material and void area (pore). Analysis of image is used to calculate total porosity and interconnectivity. It also, provides information for each pore and its pore size instead of detecting by SEM. In CAD system, edge detection for input image is available with different techniques. Scaffold was trimmed to approximately $10 \mathrm{~mm} \quad \mathrm{x} 10 \mathrm{~mm} \quad \mathrm{x} 15 \mathrm{~mm}$ using Buehler Isomet low speed saw. Thickness of sample does not affect on porosity, since we calculate surface porosity for each image and foreground objects in it.

\subsubsection{Image processing}

Thresholding is the first step in image analysis. Threshold-value is used to differentiate between foreground and background. Thresholding segments the input image into pores (void area) and scaffold materials. Small pores arising at the surface as a result of segmentation are removed automatically by erosion without affecting the bulk pore structure. Pore boundaries were detected and objects that are close to each other are connected. Subtracting from the background gives edges and area of foreground objects. By filling process, area of pores is easily detected and eliminated small dark pixels in pores. Each region in the resulting image is labelled to extract the parameters for all pores in image. Fig. 3 illustrates the process for foreground detection.

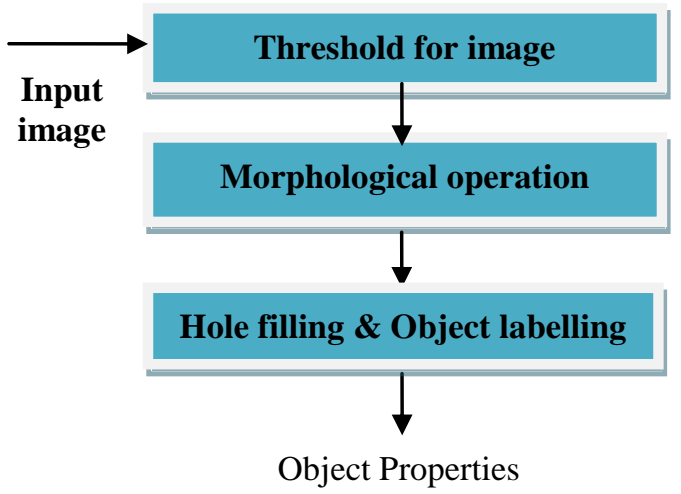

Figure 3. Summary for Image analysis technique.

\subsubsection{Interconnectivity of Pores}

In the calculation of the interconnectivity of the pore spaces, the threshold was used. The area of the interconnected spaces could be calculated. 
According to method of capture image, interconnectivity is calculated.

For longitudinal images in scaffold, the area of interconnected pores was divided by the total area of the pore spaces, thus yielding the degree of interconnectivity. The algorithm uses a combination of morphological operations applied to binaries images. By dilation operation, the connected pore regions that are more than defined area of pore are detected.

For transverse sections, interconnectivity is calculated by subtracting two consecutive images after detection of pores in each image. One minus white area divided by area of pores in first image represents the connectivity. Fig. 4 Shows steps of determination interconnectivity in case of transverse both and longitudinal sections.

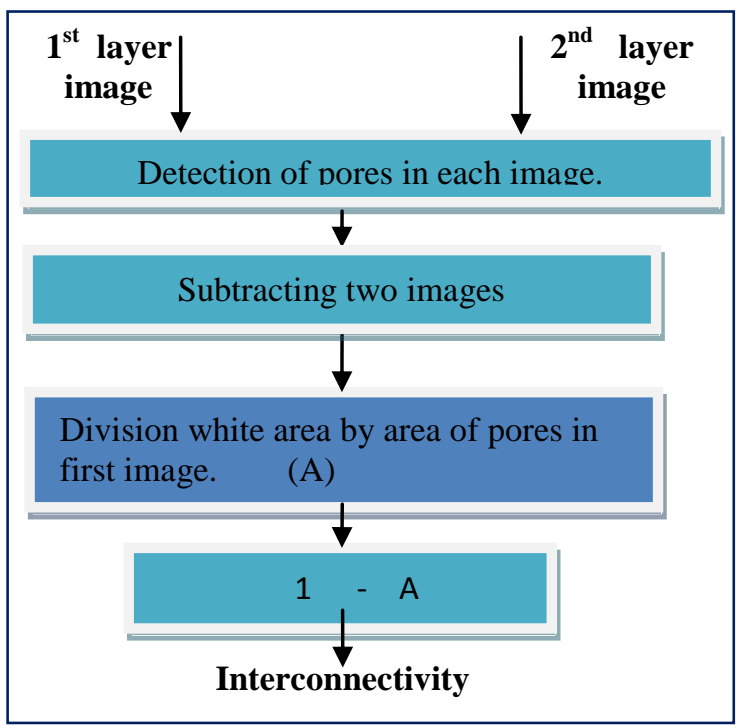

Figure 4a. Interconnectivity using transverse sections.

\subsubsection{Biodegradation Test}

Biodegradation test of porous scaffold was done using Archimedes principle. Biodegradation test of porous scaffolds was done by soaking in Tris-HCl buffer solution for one to four weeks then the samples were dried. Weight of sample after soaking in Tris- $\mathrm{HCl}$ solution was calculated after each week. The result was compared to that calculated by CAD system to verify the accuracy of the developed program and show that it could be used to calculate degradation rate.

From imaging of scaffold material before treatment in simulated body fluid and after treatment, degradation is calculated. Threshold is used to detect area of scaffold material in image. The difference area of the two images is divided by native area of scaffold material in first image. This degradation could be measured for different treatment time and curve indicated rate of degradation is plotted.

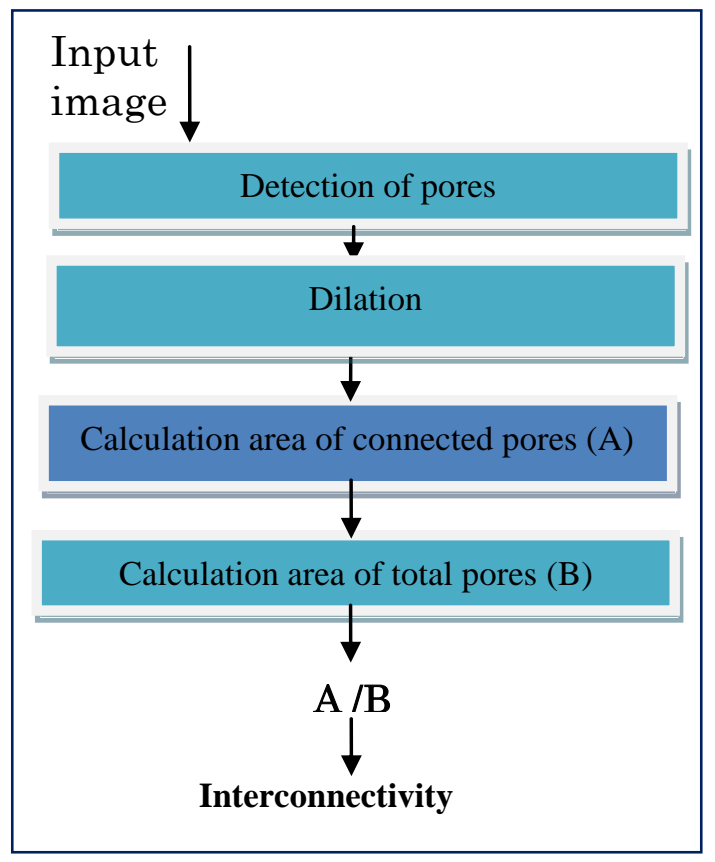

Figure 4b. Interconnectivity using longitudinal section

\subsection{Cell Isolation and Culture}

Samples of blood were taken from cord blood. $30 \mathrm{ml}$ for sample aspirated from cord blood.

The cord blood aspirated was diluted with phosphate buffer saline (PBS). $15 \mathrm{ml}$ of Ficoll to the aspirated sample was added and mononuclear cells (MNCs) were separated. Cell centrifuged at $1500 \mathrm{rpm}$ for 10 minutes to form a cell pellet. The pellet was then resuspended in fresh medium Alpha modified Eagle's medium (Alpha MEM). The mononuclear cells plated in and cultured at $37^{\circ} \mathrm{C}$ in $5 \% \mathrm{CO} 2$ incubator.

The scaffolds were seeded with cells by dispensing the cell suspension directly on their surfaces. The seeded scaffolds were incubated at $37^{\circ} \mathrm{C}$ and $5 \% \quad \mathrm{CO}_{2}$ in a humidified incubator. Scaffolds were microscopically evaluated three and eight days after seeding using SEM and Phase contrast microscope. 


\section{Material Characterization}

\subsection{Nano-particles Images}

The prepared particles were imaged by transmission electron microscope. HA particles were less than 50nm as shown in Fig.5a. For TCP, the particles as shown in Fig.5b were in range of 200 to $300 \mathrm{~nm}$.

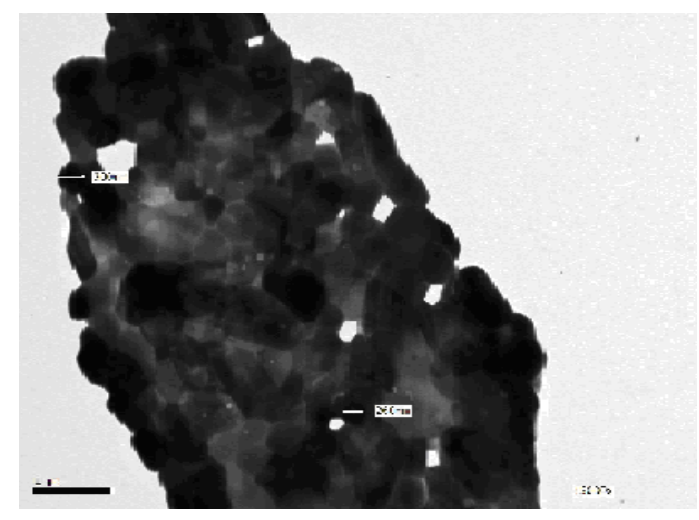

Figure 5a: TEM images for HA

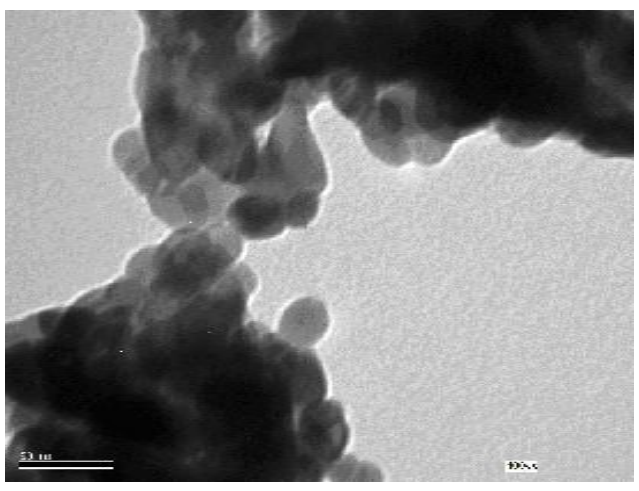

Figure 5b: TEM images for TCP.

\subsection{Polymeric sponge method}

Scanning electron microscopy shows the morphology of the scaffold using sponge method. Images show high porosity. Pore size' range is from 50 to 600 micrometer as shown in Fig. 6. A wide range of pore diameters could be seen within the same SEM image.

\subsection{Porosity Calculation Based on Image Analysis}

Porosity is the simplest property characterizing a porous medium. Porosity is the fraction of void space within the sample. After estimating the area of the scaffold within the ROI, the area of empty space is obtained. The ratio of white area to the total area of the image represents the porosity $[7,8]$, i.e. dividing the area of the void empty space by the area of the ROI gave porosity. Determination of surface porosity using image analysis is more accurate than determination using Archimedes' principle. The porosity obtained by image processing is around the measured value by any other methods as shown in Fig. 7. The comparison was done to confirm the validity of the software. It shows that the calculated porosity by CAD is greater than it by Archimedes. This may be due to ability of detecting small pores in case of using image.

Calculating porosity using Archimedes' principle shows that it increases as powder concentration decreases. Porosity of scaffold was in range of 34 to 55 at surface.
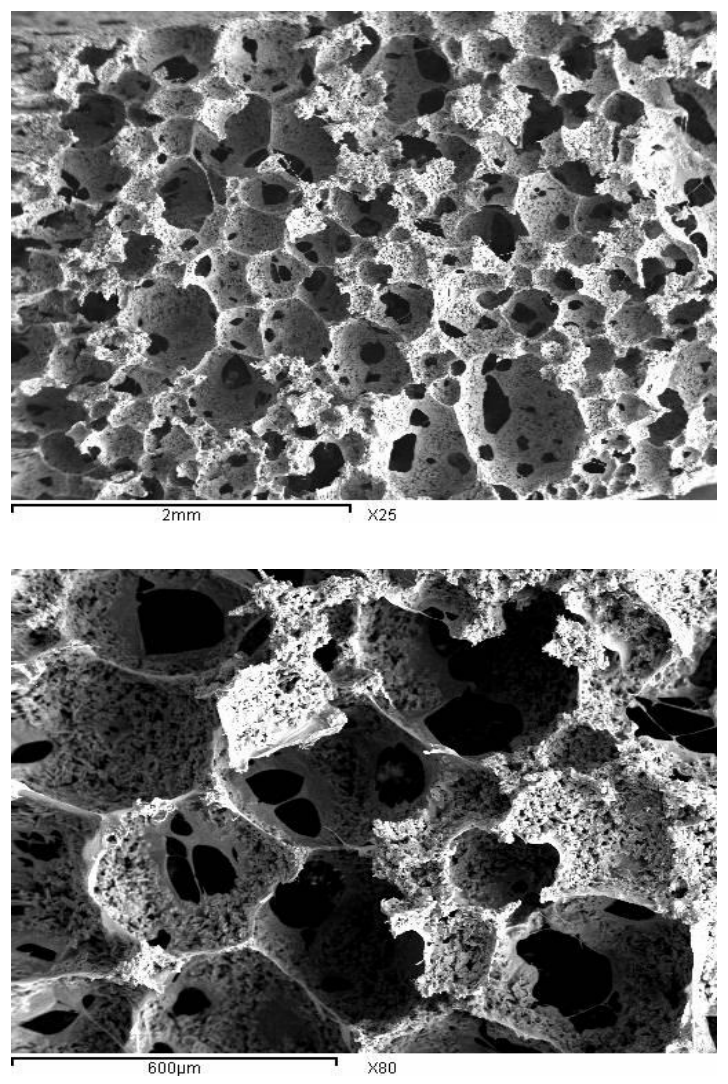

Figure 6. Cross-sectional SEM micrograph of a scaffold using sponge method with $15 \%$ powder concentration.

It is found that porosity of scaffold doesn't change significantly with particle concentration increases from 15 to $20 \%$. But, it is more porosity with concentration 10 than it in $15 \%$ 
concentration. Pore size is different as powder concentration changed. It decreased nearly by 25 to 80 micrometer as powder concentration increases.

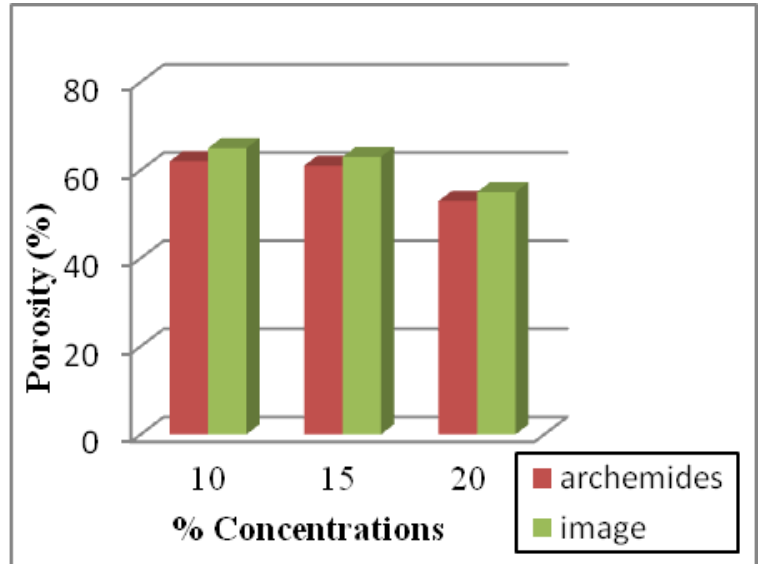

Figure 7. Porosity using image and archimedes principles.
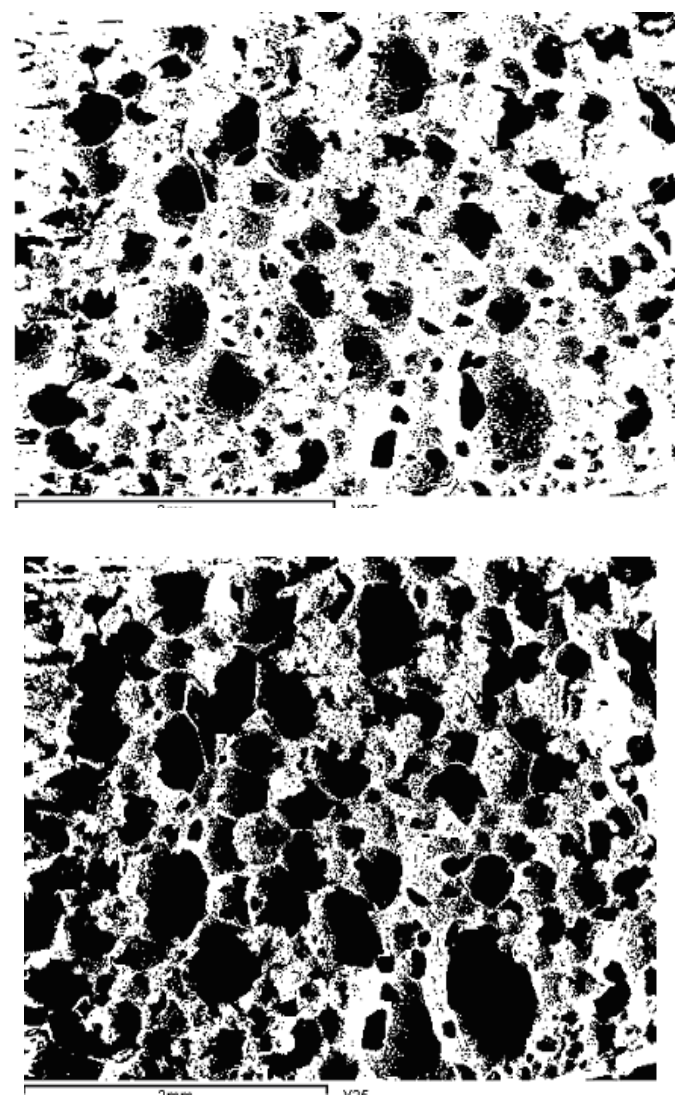

Figure 8. Effect of threshold value in detection objects

\subsubsection{Interconnectivity}

Scaffolds show good connectivity. First all pores are detected using threshold and dilation. Sum of pores area is calculated. Pores with size more than 100 micrometers are calculated. The calculated parameter (interconnectivity) was ranged from $80 \%$ to $90 \%$. The interconnectivity not affected by changing powder concentration as determined in other studied [9]. It is found that threshold value is important for success of analysis. Fig. 8 shows the effect of changing threshold value from 0.3 to 0.5 .

\subsubsection{Morphological Process}

Close operation detected edges of foreground objects and connected objects that are close to each other. Background subtraction with SE of 25 was shown in Fig. 9-a. Background subtraction and filling holes result in detection of foreground objects as shown in Fig. 9-b, it is seen that the algorithms success in defining pores.

\subsection{Mechanical Test}

Samples of scaffold were tested and the mean value of the compressive strength of scaffolds with different powder ratio was measured. The average data of the test results are plotted in the Fig.10.

Compression strength increases with decreasing the porosity and increasing powder concentration. It was up to $20 \mathrm{MPa}$. These values were suitable and similar to those for bone. It is found that as powder concentration increases, compression strength increases. In comparison to scaffolds using other methods, it is indicated that sponge method using the controlled temperature has higher compression strength. This is due to polymer used and controlled temperature. The roughness surface for scaffold was measured. It showed high roughness values compared to other materials. This indicated that cells will have high adhesion degree to scaffold surfaces. Roughness increased as nanoparticles concentration increased. It was inversely with CMC concentration.

\subsection{Biodegradation Test}

Samples showed a significant increase in the mean weight loss percent throughout all week. All three samples $\left(s_{1}, s_{2}, s_{3}\right)$ had high weight loss percent during the first week (ranging from 35\% to $40 \%$ ). However, the weight loss slowed down in the next weeks as shown in Fig. 11. Using 
images, high loss in scaffold material was found in first week. This result indicates that sampled still do its function and give time for cell to differentiate up to 28 days.
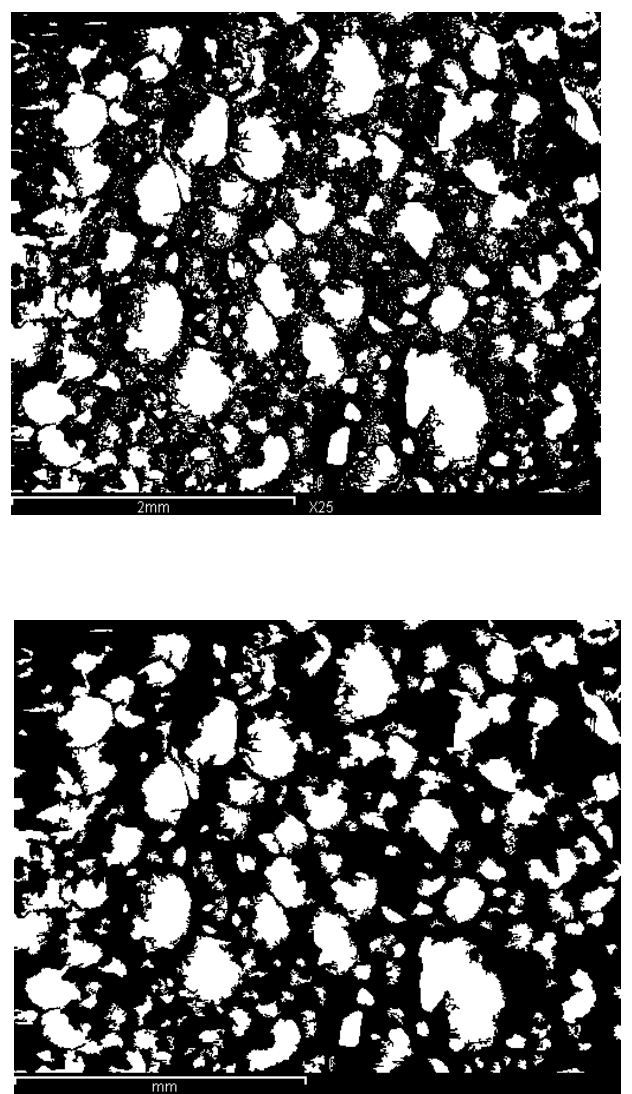

Figure 9. Subtraction background and Fill pores for detection of foreground objects.

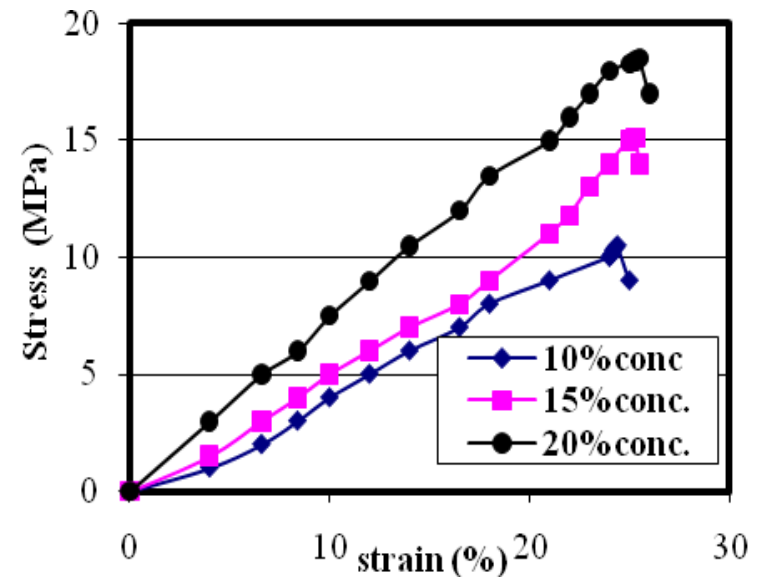

Figure 10. Compression and powder concentration.

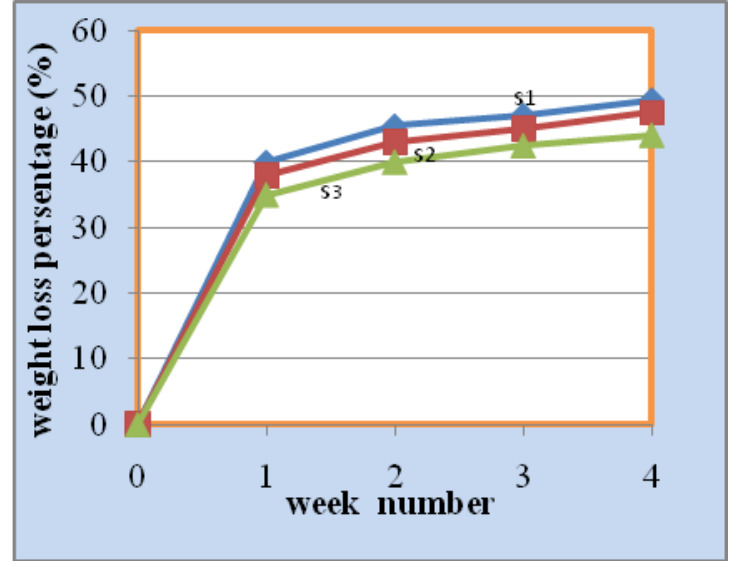

Figure 11. Mean weight loss for three samples at the end of each week.

\subsection{Cell differentiation}

The cells were used for scaffold seeding after they differentiated in culture and reached near confluence. Scaffold was imaged using scanning electron microscopes. The images showed that the osteoblastes cells adhered on the scaffolds' surfaces. The images show good differentiation and growth as shown in Fig.12. It shows cell growth on scaffold surface after 7days. Cell stretch itself across pore and cover the surface.
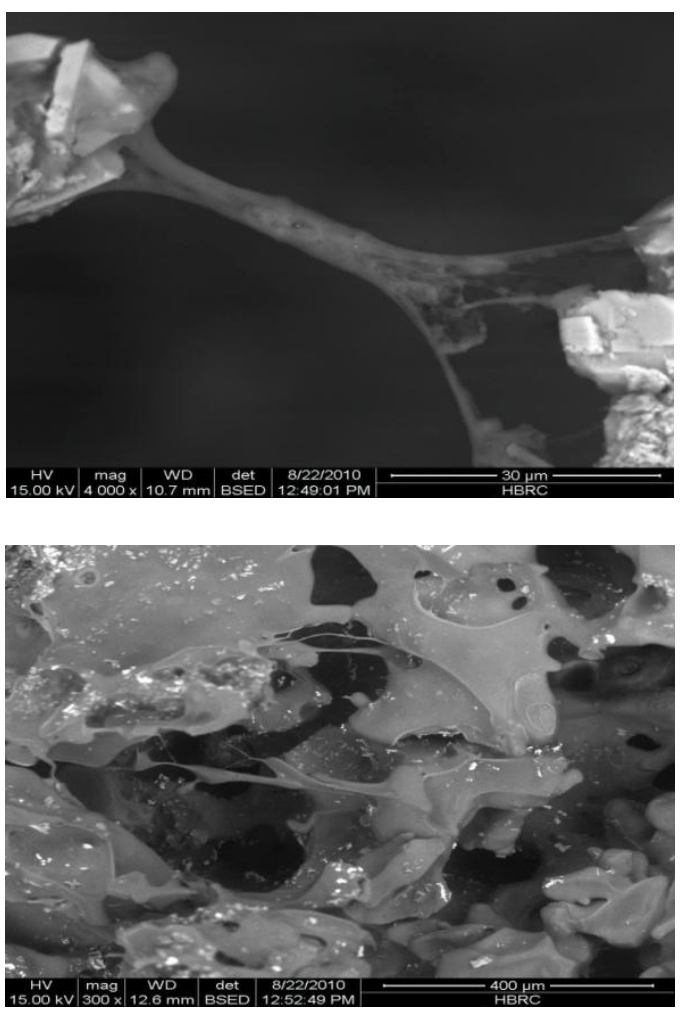

Figure 12. SEM micrographs show cell growth on scaffold surface after 7days. 


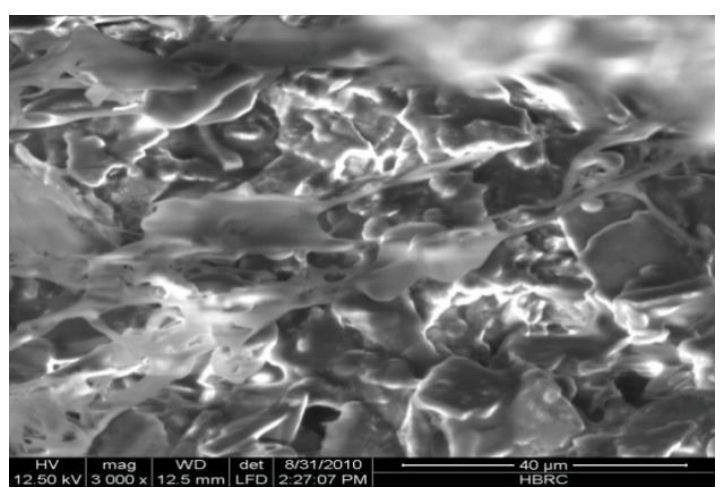

Figure 13. SEM micrographs of the scaffold surface shows cell growth after 14 days.

These results indicated that the implemented scaffold is biocompatible. Scaffold allows cell adhesion and differentiation.
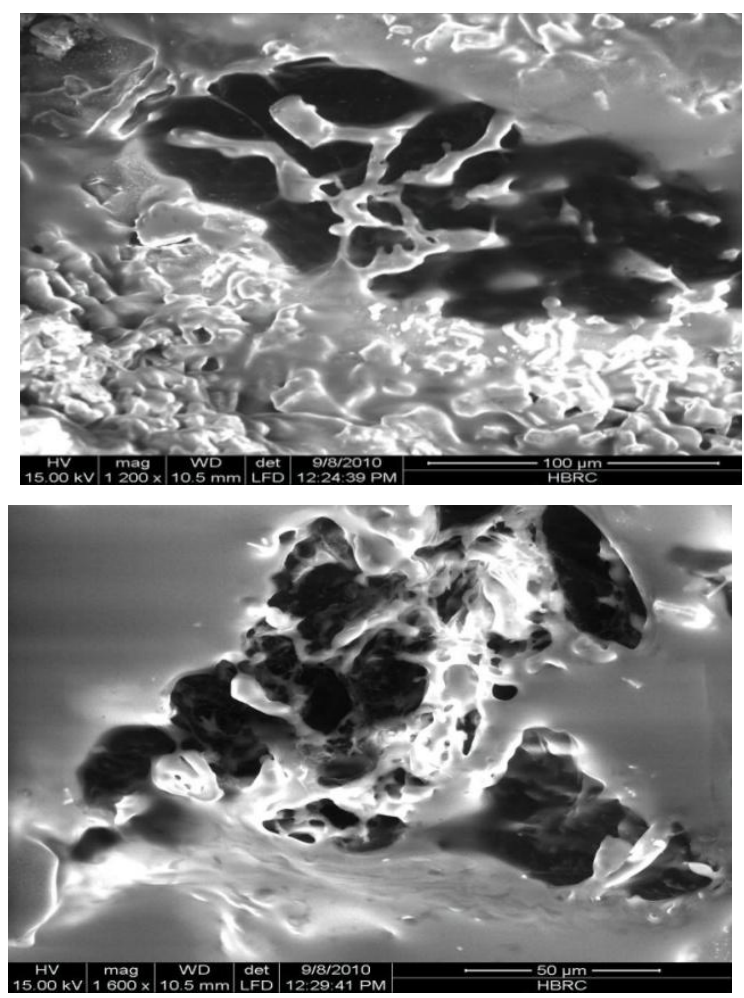

Figure 14. SEM micrographs of scaffold-cell constructs after seeding showing the attached cells on the pore wall of the scaffolds [a] and cells filling up the open pore by stretching themselves across the pore.[b].

After two weeks of seeding, the cells were differentiated inside scaffolds' pores and move out of pores. Cells continue differentiation as shown in Fig.13. The image of the scaffold surface shows cell growth with high dense after
14 days. The cells penetrate into the scaffold and migrate. The cells also attached to and colonized the walls of the scaffolds' pores as shown in Fig. 14. The cells not only lined the walls, but also stretched themselves across pores and tunnels as shown in Fig. 14.b. Some areas of the scaffolds' surfaces became covered with a layer of reticular deposits. After 28 days the scaffold was nearly full degradable and structure like bony architecture was formed as shown in Fig.15.

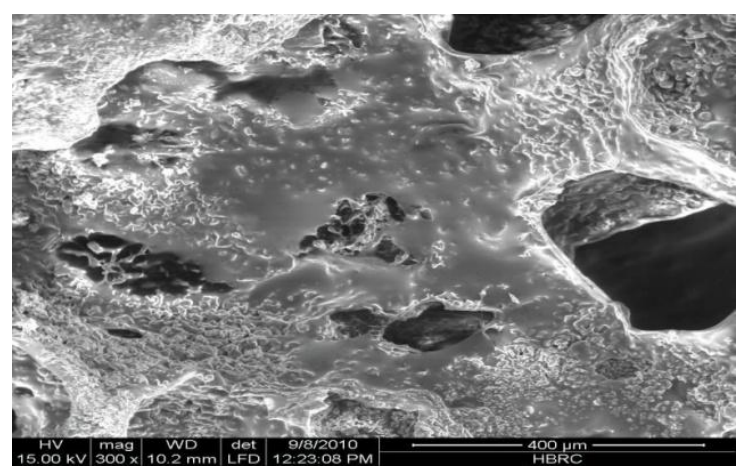

Figure 15. SEM micrograph shows bony structure.

\section{Summary}

Three scaffolds were prepared containing the same concentrations of PVA but different ratios of nanoparticles. The ratio was $10-15$ and $20 \%$. $\beta$-TCP to HA ratio was 2:3. Same CMC concentrations were added to each group. The scaffolds were imaged using scanning electron microscope (SEM). Segmentation algorithm with several techniques is used to determine parameters. Morphology analysis has been developed to determine the pore distribution and porosity of porous structure. The system allows user to define and change the used threshold value. Evaluation of implemented scaffold using CAD system is simple and fast. It doesn't need user experience as it draws boundaries.

The compressive strengths of the scaffolds were measured by compressing samples to failure using a mechanical testing machine. High compressive strengths in scaffold with high powder concentration are observed. In co-relation with porosity, compressive strengths increase as porosity decrease. It also shows that compressive 
strengths increase as CMC concentration increases. The degradation rate of the scaffolds was evaluated by comparing the scaffolds' dry weights before and after immersion in a simulated body fluid. Image system is used to define degradation of scaffold. Scaffolds were scanned before and after treatment in simulated body fluid. Image technique was used to compute difference between the two images, so degradation rate is computed. The results showed that all scaffold groups experienced rapid weight loss during the first week which slowed down thereafter. Increasing in $\mathrm{CMC}$ and ceramic concentration decreases the scaffold's degradation rate.

\section{Conclusions}

Using a new treatment process for implementing a scaffold by Polymeric Sponge Method produces high porous scaffold with high strength. These scaffolds are suitable for cartilage and bone tissue engineering. Using developed computer system permits scaffold characterization. Based on the obtained results, the prepared composite scaffolds overcame the disadvantage of using HA or TCP only. Composite based on using the two ceramic powders together allows controlling of degradation and mechanical strength. Adding CMC increased the mechanical properties and resulted in a controllable degradation rate. But, it decrease roughness value. The scaffolds porosity ranged from 30 to $60 \%$ which is similar to that in part of bone. The scaffolds have a highly porous interconnected microstructure. The increase in the powder concentration in scaffolds decreases their pore size. The increase in the powder concentration increases the compressive strength of the scaffolds. The degradation rate of the scaffold varies inversely with the $\mathrm{CMC}$ and powder concentration. The HA/ $\beta$-GP/HEC scaffolds are biocompatible since they allow the seeded cells and also encourage cell attachment and differentiation.

The developed image analysis system (CAD) is easy system and fast evaluation for any porous material. It computes porosity, interconnection directly from image. Measuring pore size is allowed without need scan system of SEM instrument. Using computer system characterises porous scaffold before and after seeding cells. It could be used with other system as finite element to provide complete evaluation, i.e. structure and mechanical properties without need mechanical tests. The SEM may yield digital images with suitable quality for further analysis. The acquisition of numerical information from these high quality images requires good and effective processing and analysis routines. Using successive stages of erosion/dilation has revealed information about the porosity, connectivity of pore structure and allowed for the estimation of pore sizes.

\section{References}

1. D. Mohn, S.K.Misra, T.J. Brunner, A.R. Boccaccini, W.J. Stark, Nano- versus Micronsized Bioactive Glass Reinforcement of P(3HB) - Are Nano-fillers the Way Forward, European Cells and Materials, Vol. 16., (page 8), ISSN 1473-2262, 2008.

2. Hirotaka Maedaa, Toshihiro Kasugaa, Masayuki Nogamia, Minoru Ueda, Preparation of bonelike apatite composite for tissue engineering scaffold, Science and Technology of Advanced Materials 6 (2005) 48-53. DOI: 10.1016/j.stam.2004.07.003

3. Zhihong Dong, Yubao Li, Qin Zou, Degradation and biocompatibility of porous nano-hydroxyapatite/polyurethane composite scaffold for bone tissue engineering, Applied Surface Science 255 (2009) 6087-6091. DOI: 10.1016/j.apsusc.2009.01.083

4. Wang M, "Developing bioactive composite materials for tissue replacement," Biomaterials, vol.24, pp. 2133-2151, 2003. DOI: 10.1016/S0142-9612(03)00037-1

5. Liuyun Jiang, Yubao Li, Xuejiang Wang, Li Zhang, Jiqiu Wen, Mei Gong,Preparation and properties of nano-hydroxyapatite/chitosan/ carboxymethyl cellulose composite scaffold, Carbohydrate Polymers 74 (2008) 680-684. DOI: 10.1016/j.carbpol.2008.04.035

6. W. Sun, B. Starly, J. Nam, A. Darling, BioCAD modeling and its applications in computer-aided tissue engineering, Computer- 
Aided Design 37 1097-1114, (2005). DOI: 10.1016/j.cad.2005.02.002

7. Lin C L, Miller J D., "Network analysis of filter cake pore structure by high resolution Xray microtomography," J. Chemical Engineering Journal, vol. 77-7, pp. 79-86, 2000.

8. Coskun S B, Wardlaw N C., "Influences of pore geometry, porosity and permeability on initial water saturation: an empirical method for estimating initial water saturation by image analysis, ”J. Petroleum Science and
Engineering, vol. 12-4, pp. 295-308, 1995. DOI: 10.1016/0920-4105(94)00051-5

9. R.Ziel, A. Haus, A. Tulke, "Quantification of the pore size distribution (porosity profiles) in microfiltration membranes by SEM, TEM and computer image analysis," Journal of membrane science, vol. 323, pp. 241-246, 2008. DOI: 10.1016/j.memsci.2008.05.057 